\title{
12. SEDIMENTARY FACIES AND SEDIMENT COMPOSITION CHANGES IN RESPONSE TO TECTONICS OF THE CÔTE D'IVOIRE-GHANA TRANSFORM MARGIN' ${ }^{1}$
}

\author{
Kari Strand ${ }^{2}$
}

\begin{abstract}
During Ocean Drilling Program Leg 159 four sites were drilled along the Côte d'Ivoire-Ghana Transform Margin (Sites 959-962), all located on a marginal ridge that defines the continental margin along a transform boundary. The Cretaceous rift to transform margin deposits document the early evolution of an active transform margin and its transition to a passive margin, thus defining a second-order, tectono-eustatic cycle of 10-50 m.y. duration. Emphasis in this paper is on sedimentary facies, sediment composition changes, and tectono-eustatic cycles in a rift/transform margin transition In the late Albian, restricted intracontinental sedimentation was characterized by laminated lacustrine and mixed-source deltaic deposits. This was followed by a progressive marine transgression with the deposition of horizontally laminated to cross-laminated, storm-influenced, shallow-shelf sandstones and claystones that terminate in a Turonian unconformity, thus defining one total third-order (1-10 Ma) tectono-eustatic cycle. The overlying periplatformal carbonate deposits of Turonian to Coniacian age were derived from shallow reefs by gravity-induced processes and from suspension, and indicate the beginning of a marginal ridge uplift. A significant siliciclastic component with lithic fragments, accompanied by carbonate sedimentation, indicates mixed, reworked sediment sources due to supposed further uplift of the marginal ridge. This uplift reduced sediment accommodation space and caused termination of this next third-order cycle during the late Coniacian-Santonian. Origin of the uplift is thought to be related to the thermal effect of the passage of a spreading ridge south of the margin. The next cycle, overlying the sandy limestones, is recorded by a condensed section of claystones, containing phosphatic debris and hardgrounds deposited over a time period of more than $10 \mathrm{~m}$.y. In a deeper setting, this condensed interval is overlain by a sequence of organic-rich black claystones, whose deposition was promoted by diminished accommodation space and partial damming of the basin by the marginal ridge during late Coniacian to early Eocene. The dominant tectonic setting shifted closer to a passive margin at each of the four drill sites after the late Paleocene.
\end{abstract}

\section{INTRODUCTION}

Drilling during Leg 159 represented the first application of deepsea drilling to the tectonic study of transform-margin development (Mascle, Lohmann, Clift, et al., 1996). Sites 959 through 962 were drilled on the Côte d'Ivoire-Ghana Marginal Ridge, a prominent marginal ridge that defines the continental margin along a fossil transform boundary. In this work, sedimentary facies, processes, and composition changes are studied in response to development of a transform continental margin with an emphasis on the rift/transform transition.

Facies analysis is integrated with provenance analysis to achieve a better understanding of source-rock composition, weathering in the source area, transport to the site of deposition, and the depositional environments. This integrated picture forms the basis on which the sedimentary indicators of the tectonic evolution of the continental margin along a transform boundary are determined. The sedimentary record found at each of the four drill sites provides a good framework for reconstructing the continental breakup history of a margin created by transform tectonics. This paper also contains the first discussions of the tectono-eustatic controls of relative change of sea level in a margin created by transform tectonics.

\section{REGIONAL AND GEOLOGIC SETTING}

The drill sites were selected to sample the sedimentary column especially from the rift to transform margin sediments of the Cretaceous. Sites 959 and 960 provide a record of development of the main

${ }^{1}$ Mascle, J., Lohmann, G.P., and Moullade, M. (Eds.), 1998. Proc. ODP, Sci. Results, 159: College Station, TX (Ocean Drilling Program).

${ }^{2}$ Department of Geology, Institute of Geosciences, University of Oulu, FIN-90570 Oulu, Finland. kari.strand@oulu.fi part of the marginal ridge, and also the most complete sedimentary record (Figs. 1, 2). Site 959 lies on the continental slope off the southwest coast of Ghana at a water depth of $2090.7 \mathrm{~m}$. Site 960 lies south of Site 959 at a water depth of $2061 \mathrm{~m}$ on the summit of the marginal ridge. By contrast, Site 961 lies on a tilted fault block on an area of thin continental crust west-southwest of Site 960 (Fig. 1). Site 962 is located on a minor ridge close to the ocean/continent transition (Fig. 1), whose acoustic basement was unknown, but had been compared to blocks of continental crust found over $300 \mathrm{~km}$ farther west along

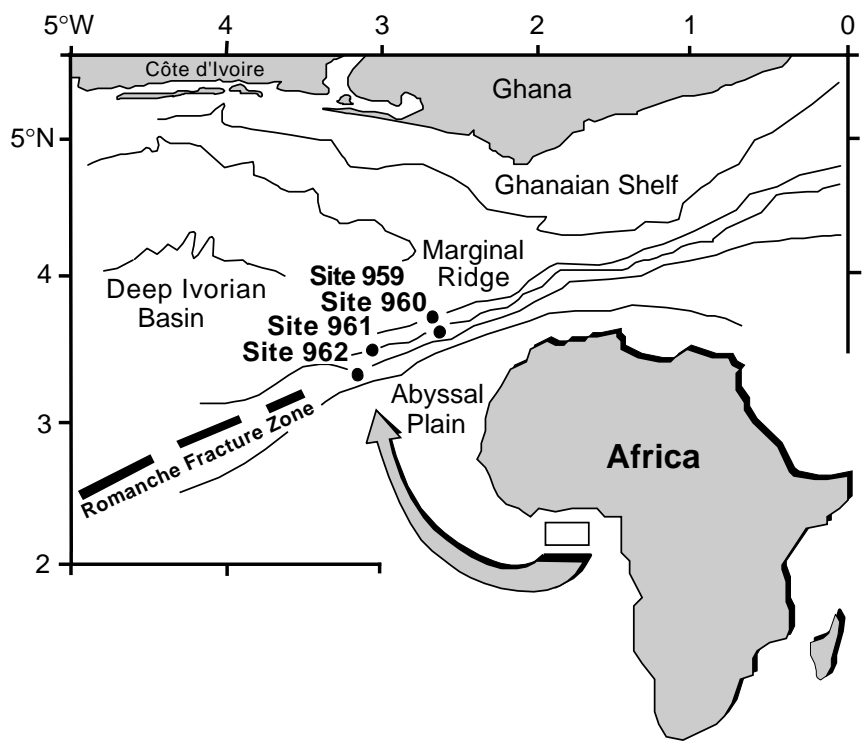

Figure 1. Location of Leg 159 sites on a simplified bathymetric map of the Côte d'Ivoire-Ghana Marginal Ridge and surroundings. 


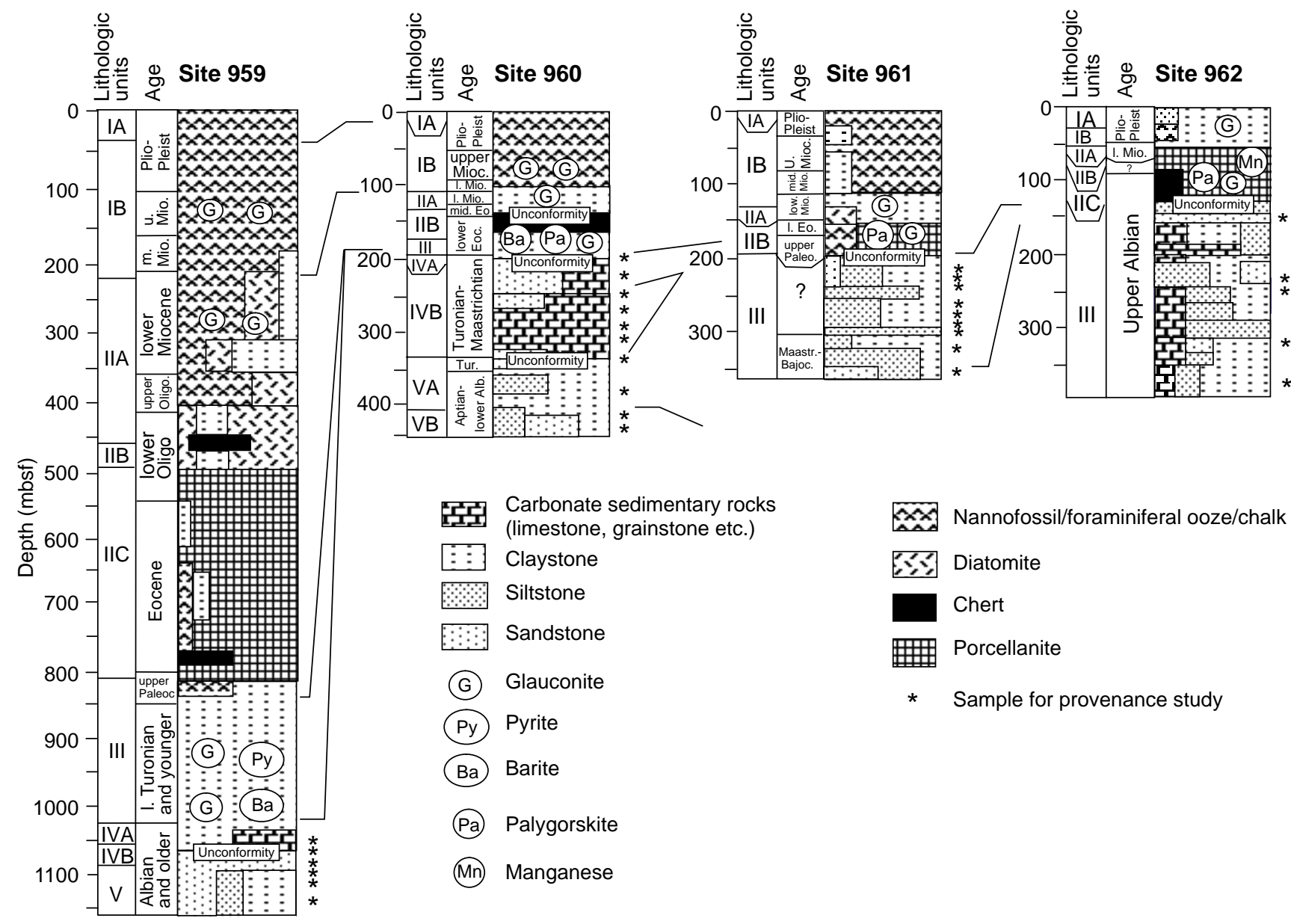

Figure 2. Schematic stratigraphic columns for Leg 159 sites showing sample intervals. Lithologic units of each site are those determined by Shipboard Scientific Party (1996).

the Romanche Fracture Zone; the blocks are believed to have been displaced there by shear tectonics (Honnorez et al., 1994). All four sites drilled during Leg 159 were located on continental crust along a ridge that runs close to the continent/ocean transition.

Basile et al. (1992, 1993), following Mascle and Blarez (1987), have proposed a four-stage schematic evolution of the Côte d'IvoireGhana Transform Margin: (1) early rifting of the Deep Ivorian Basin and shearing of its southern border during early Cretaceous time before complete breakup. Data from deep dives (Mascle et al., 1993) demonstrate that the sedimentation was subaerial, deltaic, and lacustrine during that time; (2) end of rifting and intracontinental transform faulting sometime during the Albian when initiation of seafloor spreading increased transform-type displacement between the two continental borderlands; (3) continent/ocean transform faulting in Santonian time, finally causing continental separation between West Africa and northeast Brazil, which brought into contact the newly created Gulf of Guinea oceanic crust with an active continental transform margin and manifested a complex uplift and subsidence history of the marginal ridge; and (4) subsequent passive margin evolution started during the late Paleocene, when active tectonism along the transform margin progressively ceased.

\section{MATERIALS AND METHODS}

The sedimentation on the Côte d'Ivoire-Ghana Transform Margin is best represented in the spatial and stratigraphic variation of sediments cored at Sites 959 and 960 . Although important information is provided by cores from Sites 961 and 962, the completeness of these sections, and thus the direct contribution for interpreting paleoenvironmental settings, is comparatively limited (Shipboard Scientific Party, 1996). Trends in changes of lithology, sedimentary structures, and grain size were used to identify lithofacies (Table 1), to trace the vertical character of sediment sequences, and to determine depositional environments. A total of 272 sediment samples were taken from all the cores recovered, and 56 thin sections were made from the sandy sediments. Unconsolidated samples were dried and epoxied before making thin sections. Thirty thin sections for provenance study were stained for both calcium and potassium feldspar using a method outlined in Friedman (1971). A total of 500 points were counted on each thin section using the Gazzi-Dickinson method, which minimizes the effects of grain-size variation on composition and is uniform also for poorly sorted sandy sediments (Dickinson, 1970; Ingersoll et al., 1984). Primarily, with this method, monomineralic crystals and other grains of sand size that occur within larger rock fragments are classified in the category of the crystal or other grain, rather than in the category of the larger rock fragment. Thus, plutonic lithic fragments are counted as their constituent crystals. Counted grains were placed into 12 compositional categories (Table 2 ). The biogenic category (Bio) includes molluscan fragments, echinoderm debris, foraminifers, and other bioclasts, as well as the carbonate minerals category $(\mathrm{C})$, which includes only individual carbonate grains larger than $0.063 \mathrm{~mm}$. Also, some very quartz-sand-rich carbonate sediments (Pl. 1, Figs. 1-3; Table 1, Samples 159-959D69R-1, 52-55 cm, 159-960A-25R-2, 12-14 cm, 159-960A-29R-3, 99-102 cm, 159-960A-31R-1, 35-37 cm, 159-960A-34R-1, 45-49 
Table 1. Summary of sedimentary facies types used for Leg 159 sediments.

\begin{tabular}{|c|c|c|}
\hline Facies & Characteristics & Interpretation \\
\hline \multicolumn{3}{|l|}{ Siliciclastics: } \\
\hline Facies 1: Cross-laminated sandstone & $\begin{array}{l}\text { Trough to planar cross-lamination (sometimes calcite } \\
\text { cemented); set thicknesses } 5-10 \mathrm{~cm}\end{array}$ & $\begin{array}{l}\text { Dune and sandwich bedform, reworking of } \\
\text { sands by traction currents }{ }^{\mathrm{a}}\end{array}$ \\
\hline Facies 2: Hummocky cross-bedded sandstone & $\begin{array}{l}\text { Curved, low-angle }\left(<10^{\circ}-15^{\circ}\right) \text { cross-lamination, sometimes } \\
\text { swaley cross-lamination; laminae thicknesses } 1-5 \mathrm{~mm}\end{array}$ & $\begin{array}{l}\text { Wave-generated oscillatory and combined } \\
\text { flow }^{\mathrm{b}}\end{array}$ \\
\hline Facies 3: Laminated sandstone/siltstone & Sometimes convoluted or showing small-scale ripples & $\begin{array}{l}\text { Planar beds (lower flow regime), small-scale } \\
\text { ripples formed by wave or current action }{ }^{\mathrm{a}}\end{array}$ \\
\hline Facies 4: Gravel/conglomerate & $\begin{array}{l}\text { Matrix-supported to clast-supported, usually mudstone } \\
\text { intraclastic }\end{array}$ & Debris flow ${ }^{\mathrm{a}}$ \\
\hline Facies 5: Graded to massive sand & $\begin{array}{l}\text { Thin beds of poorly sorted sand or sandstone (sometimes calcite } \\
\text { cemented) }\end{array}$ & High-density turbidity current or grain flow ${ }^{\mathrm{a}}$ \\
\hline Facies 6: Graded sand-silt couplet & $\begin{array}{l}\text { Couplet composed of a basal sandy unit and massive to } \\
\text { laminated fine-grained sediment; bed thickness } 1-5 \mathrm{~cm} \text {, some } \\
\text { load casts (sometimes with micrite) }\end{array}$ & Low-density turbidity current ${ }^{\mathrm{a}, \mathrm{c}}$ \\
\hline $\begin{array}{l}\text { Facies 7: Laminated to massive silty claytone to } \\
\text { clayey siltstone }\end{array}$ & $\begin{array}{l}\text { Fine-grained sediment showing layering and thin silt-rich } \\
\text { horizontal laminae, some } 1-2 \mathrm{~mm} \text { thick, irregular lamination } \\
\text { or structures (sometimes calcite cemented) }\end{array}$ & $\begin{array}{l}\text { Fine-grained or mud turbidity current } \mathrm{t}^{\mathrm{a}, \mathrm{c}, \mathrm{d}} \text { or } \\
\text { winnowed lamination from reworking by } \\
\text { bottom-current }{ }^{\mathrm{b}}\end{array}$ \\
\hline Facies 8: Silty claystone to claystone & $\begin{array}{l}\text { Fine-grained sediments usually with various proportions of } \\
\text { siliceous and calcareous microfossils; locally bioturbated }\end{array}$ & Hemipelagic sedimentation $^{\mathrm{a}}$ \\
\hline \multicolumn{3}{|l|}{ Carbonates: } \\
\hline Facies 9: Skeletal packstone to wackestone & Massive, crudely bedded, skeletal debris & Debris flows ${ }^{\mathrm{e}}$ \\
\hline Facies 10: Graded skeletal grainstone & $\begin{array}{l}\text { Massive or thick horizontal bedding, sometimes mudstone } \\
\text { intraclastic }\end{array}$ & High-density turbidity current or grain flow ${ }^{e}, f$ \\
\hline $\begin{array}{l}\text { Facies 11: Graded to massive limestone with } \\
\text { quartz sand }\end{array}$ & Thin beds of poorly sorted carbonate debris and sand & High-density turbidity current ${ }^{e}$ \\
\hline Facies 12: Graded limestone-silt couplet & $\begin{array}{l}\text { Couplet composed of a basal limestone unit (containing some } \\
\text { quartz sand) and massive to laminated fine-grained sediment, } \\
\text { sometimes showing isolated ripple, bed thickness } 1-2 \mathrm{~cm}\end{array}$ & Low-density turbidity currents ${ }^{\mathrm{e}, \mathrm{f}}$ \\
\hline Facies 13: Laminated clayey limestone & Fine-grained sediment showing fine laminae & $\begin{array}{l}\text { Fine-grained turbidity current or traction } \\
\text { transport by bottom-current } \mathrm{e}^{\mathrm{e}} \mathrm{f}\end{array}$ \\
\hline \multicolumn{3}{|l|}{ Associated biogenic facies: } \\
\hline Facies 14: Nannofossil/foraminifer ooze/chalk & Massive, mottled, sometimes clayey & Pelagic to hemipelagic sedimentation ${ }^{\mathrm{a}, \mathrm{e}}$ \\
\hline $\begin{array}{l}\text { Facies 15: Diatomaceous ooze/diatomite / } \\
\text { porcellanite }\end{array}$ & Massive, mottled, sometimes clayey & Pelagic to hemipelagic sedimentation ${ }^{\mathrm{a}}$ \\
\hline
\end{tabular}

Note: ${ }^{\mathrm{a}}=$ Pickering et al., 1986,$1989 ;{ }^{\mathrm{b}}=$ Nottredt and Kreisa, $1987 ;{ }^{\mathrm{c}}=$ Stow and Shanmugam, $1980 ;{ }^{\mathrm{d}}=$ Piper and Stow, $1991 ;{ }^{\mathrm{c}}=$ Scholle et al., $1983 ;{ }^{\mathrm{f}}=$ Tucker and Wright, 1990.

$\mathrm{cm}$, and 159-960C-25X-2, 41-44 cm) were counted. Although the carbonate minerals category accounts for a substantial fraction in these samples and the samples may not be suitable for use in ternary diagrams, important knowledge about the composition of the framework grains such as quartz, feldspars, and rock fragments could be obtained. Recalculated parameters are defined and presented in Table 3 , and also plotted on ternary diagrams showing provenance domains after Dickinson et al. (1983). In provenance discrimination, using ternary diagrams, special care is required when the number of framework grains counted is small. Thus, in this work, the source-rock evaluations are predominantly based on the careful thin-section observations, and emphasis is laid in sediment composition changes.

\section{SEDIMENTARY FACIES AND DEPOSITIONAL ENVIRONMENTS}

Sedimentary facies are described and depositional environments are interpreted within the framework of three principal evolutionary stages, mostly based on the sequences reconstructed for Sites 959 and 960 (Fig. 2): (1) Albian rift to early post-rift stage; (2) continent/ ocean transform stage during the Cenomanian to Coniacian; and (3) transform to passive margin transition during the late Coniacian to early Eocene. Fifteen sedimentary facies have been distinguished among the Leg 159 clastic deposits based on grain size, texture, and dominant sedimentary structures. The principal facies types and their interpretation are outlined in Table 1 . The selected core-face photographs and microphotographs of all major coarse-grained facies are available on CD-ROM (back pocket, this volume).

\section{Albian Rift to Early Postrift Stage}

\section{Lithofacies}

The oldest record of sedimentation recovered during Leg 159 includes the Albian successions of finely laminated, siliciclastic rhyth- mites ranging from silty sandstones to silty claystone. The following facies or facies successions were identified: (1) laminated to convoluted sandstone (Facies 3; Fig. 3A); (2) laminated siltstone (Facies 3; Fig. 3B); and (3) laminated silty claystone (Facies 7; Fig. 3C). The fine sandstone laminae in Facies 3 are up to $5 \mathrm{~mm}$ in thickness. Parallel lamination in Facies 7 appears to represent graded rhythms of very fine sand and silt some 1-2 mm thick turning to clay, occasionally interbedded with $1-2 \mathrm{~cm}$ thick, graded silty sandstone-siltstone couplets (Sample 159-960A-58R-1, 47-60 cm, on CD-ROM, back pocket, this volume). The coarsest siliciclastic sediments in these successions are dominated by fresh detrital feldspar and angular to subangular monocrystalline quartz (Pl. 1, Figs. 4, 5, Sample 159960A-46R-2, 48-51 cm, on CD-ROM). Going upsection at Site 959, finely laminated sediments are overlain by dominantly sandy sediments, consisting of hummocky cross-bedded sandstones (Facies 2; Figs. 3D, 4A, Sample 159-962D-6R-1, 8-24 cm, on CD-ROM) and horizontally laminated (Facies 3) to cross-laminated (Facies 1) sandstone beds (Fig. 3E, Sample 159-959D-73R-1, 112-126 cm, on CDROM) in laminated silty claystone (Facies 7 and 8). At Site 962, a thick upper Albian sequence consists of graded limestone with varying amount of quartz sand as framework grains (Facies 11), limestone-siltstone couplets (Facies 12), and some sandstone beds and claystones interbedded with nannofossil and micritic carbonate. Fining-upward successions show scoured bases, internal grading, and lamination. Typically, the beds consist of isolated ripples (Fig. 4B). Coarse-grained intervals are quartz-sand rich and consist of benthic and planktonic foraminifers and bivalve fragments (Pl.1, Fig. 6).

\section{Interpretation}

The oldest record recovered from Leg 159 is interpreted to represent intracontinental sedimentation, with the laminated rhythmites corresponding to lacustrine sedimentation and the laminated to convoluted sandy successions suggesting mixed-source deltaic deposition. The sediments were deposited by gravity-driven processes such 
Table 2. Counted parameters.

\begin{tabular}{|c|c|c|c|c|c|c|c|c|c|c|c|c|c|c|c|}
\hline $\begin{array}{l}\text { Core, section, } \\
\text { interval }(\mathrm{cm})\end{array}$ & $\begin{array}{l}\text { Depth } \\
\text { (mbsf) }\end{array}$ & Age & Qp & Qm & $\mathrm{P}$ & $\mathrm{K}$ & Lss & Lsc & Lv & $\mathrm{Lm}$ & $\mathrm{C}$ & M & $\mathrm{D}$ & Bio & Total \\
\hline \multicolumn{16}{|l|}{ 159-959D- } \\
\hline $69 \mathrm{R}-1,52-55$ & 1063.22 & & 6 & 135 & 1 & 2 & 3 & 2 & 0 & 10 & 193 & 0 & 3 & 145 & 500 \\
\hline 71R-1, 98-101 & 1081.88 & late Albian & 25 & 188 & 10 & 0 & 0 & 0 & 1 & 20 & 237 & 14 & 5 & 0 & 500 \\
\hline 72R-3, 108-111 & 1095.28 & & 20 & 327 & 9 & 2 & 2 & 0 & 1 & 61 & 67 & 10 & 1 & 0 & 500 \\
\hline 74R-2, 62-65 & 1112.62 & & 14 & 302 & 12 & 8 & 0 & 0 & 0 & 12 & 104 & 39 & 7 & 2 & 500 \\
\hline $76 \mathrm{R}-2,0-3$ & 1131.30 & & 39 & 328 & 19 & 3 & 9 & 0 & 5 & 39 & 34 & 23 & 1 & 0 & 500 \\
\hline 77R-2, 70-72 & 1141.70 & & 36 & 337 & 16 & 0 & 4 & 0 & 1 & 36 & 45 & 23 & 1 & 1 & 500 \\
\hline \multicolumn{16}{|l|}{ 159-960A- } \\
\hline 21R-1, 121-124 & 185.31 & San-Tur & 10 & 42 & 5 & 2 & 0 & 0 & 0 & 3 & 427 & 2 & 0 & 9 & 500 \\
\hline $25 \mathrm{R}-2,12-14$ & 223.82 & San-Tur & 9 & 47 & 5 & 1 & 4 & 0 & 0 & 2 & 215 & 0 & 0 & 217 & 500 \\
\hline 29R-3, 99-102 & 259.56 & San-Tur & 12 & 67 & 1 & 1 & 4 & 2 & 0 & 2 & 185 & 0 & 0 & 226 & 500 \\
\hline $31 \mathrm{R}-1,35-37$ & 271.45 & San-Tur & 2 & 44 & 1 & 3 & 2 & 0 & 0 & 2 & 194 & 1 & 0 & 251 & 500 \\
\hline $34 \mathrm{R}-1,45-49$ & 300.05 & San-Tur & 1 & 9 & 0 & 0 & 5 & 0 & 0 & 0 & 190 & 0 & 0 & 295 & 500 \\
\hline $37 \mathrm{R}-2,49-52$ & 329.82 & & 17 & 295 & 25 & 3 & 0 & 0 & 0 & 12 & 142 & 3 & 3 & 0 & 500 \\
\hline $46 \mathrm{R}-2,48-51$ & 373.78 & & 34 & 304 & 28 & 5 & 1 & 0 & 0 & 11 & 109 & 6 & 2 & 0 & 500 \\
\hline $57 \mathrm{R}-1,18-22$ & 427.58 & & 3 & 154 & 18 & 4 & 1 & 0 & 0 & 5 & 291 & 22 & 2 & 0 & 500 \\
\hline $61 \mathrm{R}-1,94-98$ & 447.14 & & 3 & 345 & 35 & 7 & 0 & 0 & 1 & 19 & 61 & 26 & 3 & 0 & 500 \\
\hline \multicolumn{16}{|l|}{ 159-960C- } \\
\hline $25 \mathrm{X}-2,41-44$ & 335.11 & San-Tur & 1 & 18 & 2 & 1 & 0 & 12 & 0 & 0 & 92 & 0 & 0 & 374 & 500 \\
\hline \multicolumn{16}{|l|}{ 159-961A- } \\
\hline 23R-1, 50-51 & 206.60 & & 1 & 438 & 10 & 0 & 0 & 0 & 0 & 1 & 21 & 26 & 3 & 0 & 500 \\
\hline $26 \mathrm{R}-2,44-45$ & 237.54 & & 0 & 469 & 11 & 5 & 0 & 0 & 0 & 0 & 7 & 7 & 1 & 0 & 500 \\
\hline 29R-1, 19-21 & 265.09 & & 0 & 466 & 6 & 0 & 0 & 0 & 0 & 0 & 16 & 12 & 0 & 0 & 500 \\
\hline 31R-1, 51-54 & 284.71 & & 0 & 398 & 9 & 0 & 0 & 0 & 0 & 0 & 71 & 16 & 6 & 0 & 500 \\
\hline $33 \mathrm{R}-1,17-22$ & 299.67 & & 1 & 472 & 11 & 4 & 0 & 0 & 0 & 0 & 3 & 9 & 0 & 0 & 500 \\
\hline \multicolumn{16}{|l|}{ 159-961B- } \\
\hline 2R-1, 27-29 & 226.27 & & 0 & 333 & 27 & 1 & 0 & 0 & 0 & 2 & 2 & 124 & 11 & 0 & 500 \\
\hline $5 \mathrm{R}-3,5-8$ & 270.55 & & 0 & 435 & 33 & 10 & 0 & 0 & 0 & 0 & 2 & 16 & 4 & 0 & 500 \\
\hline 13R-2, 37-39 & 333.97 & & 0 & 364 & 9 & 3 & 0 & 0 & 0 & 1 & 3 & 113 & 7 & 0 & 500 \\
\hline 18R-2, 81-85 & 364.83 & & 0 & 414 & 26 & 3 & 0 & 0 & 0 & 0 & 28 & 27 & 2 & 0 & 500 \\
\hline \multicolumn{16}{|l|}{ 159-962D- } \\
\hline 6R-1, 23-25 & 123.63 & late Albian & 2 & 409 & 16 & 7 & 0 & 0 & 0 & 28 & 36 & 1 & 1 & 0 & 500 \\
\hline 18R-4, 104-107 & 244.64 & late Albian & 1 & 351 & 14 & 9 & 1 & 1 & 0 & 5 & 95 & 1 & 1 & 21 & 500 \\
\hline 19R-1, 117-121 & 249.97 & late Albian & 0 & 323 & 8 & 6 & 6 & 0 & 0 & 4 & 137 & 9 & 3 & 4 & 500 \\
\hline 28R-1, 139-141 & 317.69 & late Albian & 1 & 268 & 8 & 6 & 0 & 0 & 0 & 0 & 159 & 16 & 2 & 40 & 500 \\
\hline 36R-1, 4-7 & 383.94 & late Albian & 0 & 96 & 5 & 1 & 0 & 0 & 0 & 2 & 356 & 0 & 1 & 39 & 500 \\
\hline
\end{tabular}

Note: $\mathrm{Qp}=$ polycrystalline quartz, $\mathrm{Qm}=$ monocrystalline quartz, $\mathrm{P}=$ plagioclase feldspar, $\mathrm{K}=$ potassium feldspar, Lss = siliciclastic sedimentary lithic fragment, Lsc = carbonate sedimentary lithic fragment, $\mathrm{Lv}=$ volcanic/metavolcanic lithic fragment, $\mathrm{Lm}=$ metamorphic lithic fragment, $\mathrm{C}=$ carbonate minerals, $\mathrm{M}=$ mica flakes, $\mathrm{D}=$ dense minerals, $\mathrm{Bio}=$ biogenic grains. San = Santonian, Tur = Turonian.

as low-density turbidity currents partially reworked by traction currents (cf. Pickering et al., 1986). The lacustrine sediments are overlain by a shallow marine shelf sequence, as indicated by hummocky cross-bedded sandstones (e.g., Nottredt and Kreisa, 1987; Myrow and Southard, 1991) and by the occurrence of cross-bedded intervals, in general, as well as by the lack of features typical of turbidity currents. All these features indicate deposition in a storm-influenced shelf at relatively low sea level. However, the Site 962 sediments record a deep and fully pelagic, marine depositional setting at the same time. Overall, the earliest sediments recovered during Leg 159 are believed to have formed in deep, tectonically generated basins associated with the transform tectonism between South America and Africa. Site 962 could represent a deep, relatively sediment-starved, pull-apart basin, such as are well known in transform settings (BenAvraham et al., 1979; Pitman et al., 1985).

\section{Continent/Ocean Transform Stage During the Cenomanian to Coniacian}

\section{Lithofacies}

The transition from the Albian early post-rift stage to the succeeding continent/ocean transform stage is marked by a Turonian unconformity (see interval 159-960A-37R-1, 36-57 cm, on CD-ROM, back pocket, this volume), at Site 960 , between laminated sandstone (Facies 3) and graded, skeletal grainstone (Facies 10). The syntransform, siliciclastic sediments below the unconformity at Sites 959 and 960 are dated as middle Turonian (Shipboard Scientific Party, 1996). At Site 960, the siliciclastic sediments include massive sandstone (Facies 5), which is characterized by sediment-filled veins (interval
159-960A-37R-2, 48-65 cm, on CD-ROM) and concentrations of mud clasts in a few centimeter-thick layers (Facies 4). However, sedimentation during this tectonic stage mostly coincides with the Turonian to Coniacian carbonates at Sites 959 and 960. The total thickness of the limestone succession at Site 959 is $\sim 40 \mathrm{~m}$, and at Site 960 is up to $140 \mathrm{~m}$. Distinguished facies are (1) massive to graded skeletal grainstone, locally containing mudstone intraclasts (Facies 10; Figs. 5A, 6A); (2) massive to crudely bedded skeletal packstone (Facies 9; Figs. 5B, 6B); and (3) laminated quartz-sand grainstone and floatstone with as much as $10 \%-20 \%$ quartz sand (Facies 11; Fig. 5C). The skeletal grainstone contains molluscan fragments and echinoderm debris and a few quartz grains (Pl. 1, Fig. 3). Pore spaces are generally filled with a calcite cement (see Sample 159-959D-69R-1, 52-55 cm, on CD-ROM). Siliciclastic components include rounded quartz grains and metamorphic, siliciclastic sedimentary and reworked carbonate sedimentary lithic fragments.

\section{Interpretation}

The sediments overlying the unconformity at Site 960 and their lateral equivalents at Site 959 are interpreted as periplatform deposits, such as debris and grain flows from a shallow shelf setting into an adjacent basin (cf. Scholle et al., 1983; Tucker and Wright, 1990). At Site 960, shallower water limestones are found. Carbonate reefs probably colonized shallow regions associated with the crest of the uplifted marginal ridge, produced by tectonic stress release along the continent/continent transform. Thus, coeval carbonate and siliciclastic sedimentation could indicate a maximum uplift of the marginal ridge. This is further evidenced by the occurrence of reworked carbonate sedimentary lithic fragments in the limestone succession. 
Table 3. Recalculated parameters.

\begin{tabular}{|c|c|c|c|c|c|c|c|c|c|c|c|}
\hline \multirow{2}{*}{$\begin{array}{l}\text { Core, section, } \\
\text { interval }(\mathrm{cm})\end{array}$} & \multirow{2}{*}{$\begin{array}{l}\text { Depth } \\
\text { (mbsf) }\end{array}$} & \multirow[b]{2}{*}{ Age } & \multicolumn{3}{|c|}{ QFL } & \multicolumn{3}{|c|}{ QmFLtot } & \multicolumn{3}{|c|}{ LmLvLs } \\
\hline & & & $\% \mathrm{Q}$ & $\% \mathrm{~F}$ & $\% \mathrm{~L}$ & $\% \mathrm{Qm}$ & $\% \mathrm{~F}$ & $\%$ Ltot & $\% \mathrm{Lm}$ & $\% \mathrm{Lv}$ & $\% \mathrm{Ls}$ \\
\hline \multicolumn{12}{|l|}{ 159-959D- } \\
\hline $69 \mathrm{R}-1,52-55$ & 1063.22 & & 88.7 & 1.9 & 9.4 & 84.9 & 1.9 & 13.2 & 66.7 & 0.0 & 33.3 \\
\hline 71R-1, 98-101 & 1081.88 & late Albian & 87.3 & 4.1 & 8.6 & 77.0 & 4.1 & 18.9 & 95.2 & 4.8 & 0.0 \\
\hline $72 \mathrm{R}-3,108-111$ & 1095.28 & & 82.2 & 2.6 & 15.2 & 77.5 & 2.6 & 19.9 & 95.3 & 1.6 & 3.1 \\
\hline $74 \mathrm{R}-2,62-65$ & 1112.62 & & 90.8 & 5.8 & 3.4 & 86.7 & 5.8 & 7.5 & 100.0 & 0.0 & 0.0 \\
\hline $76 \mathrm{R}-2,0-3$ & 1131.30 & & 83.0 & 4.9 & 12.0 & 74.2 & 4.9 & 20.9 & 73.6 & 9.4 & 17.0 \\
\hline $77 \mathrm{R}-2,70-72$ & 1141.70 & & 86.8 & 3.7 & 9.5 & 78.4 & 3.7 & 17.9 & 87.8 & 2.4 & 9.8 \\
\hline \multicolumn{12}{|l|}{$159-960 \mathrm{~A}-$} \\
\hline 21R-1, 121-124 & 185.31 & San-Tur & 83.9 & 11.3 & 4.8 & 67.7 & 11.3 & 21.0 & 100.0 & 0.0 & 0.0 \\
\hline $25 \mathrm{R}-2,12-14$ & 223.82 & San-Tur & 82.4 & 8.8 & 8.8 & 69.1 & 8.8 & 22.1 & 33.3 & 0.0 & 66.7 \\
\hline 29R-3, 99-102 & 259.56 & San-Tur & 88.8 & 2.2 & 9.0 & 75.3 & 2.2 & 22.5 & 25.0 & 0.0 & 75.0 \\
\hline $31 \mathrm{R}-1,35-37$ & 271.45 & San-Tur & 85.2 & 7.4 & 7.4 & 81.5 & 7.4 & 11.1 & 50.0 & 0.0 & 50.0 \\
\hline $34 \mathrm{R}-.1,45-49$ & 300.05 & San-Tur & 66.7 & 0.0 & 33.3 & 60.0 & 0.0 & 40.0 & 0.0 & 0.0 & 100.0 \\
\hline $37 \mathrm{R}-2,49-52$ & 329.82 & & 88.6 & 8.0 & 3.4 & 83.8 & 8.0 & 8.2 & 100.0 & 0.0 & 0.0 \\
\hline $46 \mathrm{R}-2,48-51$ & 373.78 & & 88.3 & 8.6 & 3.1 & 79.4 & 8.6 & 12.0 & 91.7 & 0.0 & 8.3 \\
\hline $57 \mathrm{R}-1,18-22$ & 427.58 & & 84.9 & 11.9 & 3.2 & 83.2 & 11.9 & 4.9 & 83.3 & 0.0 & 16.7 \\
\hline $61 \mathrm{R}-1,94-98$ & 447.14 & & 84.9 & 10.2 & 4.9 & 84.1 & 10.2 & 5.7 & 95.0 & 5.0 & 0.0 \\
\hline \multicolumn{12}{|l|}{ 159-960C- } \\
\hline $25 \mathrm{X}-2,41-44$ & 335.11 & San-Tur & 55.9 & 8.8 & 35.3 & 52.9 & 8.8 & 38.3 & 0.0 & 0.0 & 100.0 \\
\hline \multicolumn{12}{|l|}{ 159-961A- } \\
\hline 23R-1, 50-51 & 206.60 & & 97.6 & 2.2 & 0.2 & 97.3 & 2.2 & 0.5 & 100.0 & 0.0 & 0.0 \\
\hline $26 \mathrm{R}-2,44-45$ & 237.54 & & 96.7 & 3.3 & 0.0 & 96.7 & 3.3 & 0.0 & 0.0 & 0.0 & 0.0 \\
\hline 29R-1, 19-21 & 265.09 & & 98.7 & 1.3 & 0.0 & 98.7 & 1.3 & 0.0 & 0.0 & 0.0 & 0.0 \\
\hline $31 \mathrm{R}-1,51-54$ & 284.71 & & 97.7 & 2.3 & 0.0 & 97.7 & 2.3 & 0.0 & 0.0 & 0.0 & 0.0 \\
\hline $33 \mathrm{R}-1,17-22$ & 299.67 & & 96.9 & 3.1 & 0.0 & 96.7 & 3.1 & 0.2 & 0.0 & 0.0 & 0.0 \\
\hline \multicolumn{12}{|l|}{ 159-961B- } \\
\hline 2R-1, 27-29 & 226.27 & & 91.7 & 7.7 & 0.6 & 91.7 & 7.7 & 0.6 & 100.0 & 0.0 & 0.0 \\
\hline 5R-3, 5-8 & 270.55 & & 91.0 & 9.0 & 0.0 & 91.0 & 9.0 & 0.0 & 0.0 & 0.0 & 0.0 \\
\hline 13R-2, 37-39 & 333.97 & & 96.5 & 3.2 & 0.3 & 96.5 & 3.2 & 0.3 & 100.0 & 0.0 & 0.0 \\
\hline $18 \mathrm{R}-2,81-85$ & 364.83 & & 93.4 & 6.6 & 0.0 & 93.4 & 6.6 & 0.0 & 0.0 & 0.0 & 0.0 \\
\hline \multicolumn{12}{|l|}{ 159-962D- } \\
\hline $6 \mathrm{R}-1,23-25$ & 123.63 & late Albian & 88.9 & 5.0 & 6.1 & 88.5 & 5.0 & 6.5 & 100.0 & 0.0 & 0.0 \\
\hline $18 \mathrm{R}-4,104-107$ & 244.64 & late Albian & 92.1 & 6.0 & 1.9 & 91.9 & 6.0 & 2.1 & 71.4 & 0.0 & 28.6 \\
\hline $19 \mathrm{R}-1,117-121$ & 249.97 & late Albian & 93.1 & 4.0 & 2.9 & 93.1 & 4.0 & 2.9 & 40.0 & 0.0 & 60.0 \\
\hline $28 \mathrm{R}-1,139-141$ & 317.69 & late Albian & 95.1 & 4.9 & 0.0 & 94.7 & 4.9 & 0.4 & 0.0 & 0.0 & 0.0 \\
\hline $36 \mathrm{R}-1,4-7$ & 383.94 & late Albian & 92.3 & 5.8 & 1.9 & 92.3 & 5.8 & 1.9 & 100.0 & 0.0 & 0.0 \\
\hline
\end{tabular}

Note: $\mathrm{Q}=\mathrm{Qm}+\mathrm{Qp}, \mathrm{F}=\mathrm{P}+\mathrm{K}, \mathrm{L}=\mathrm{Lss}+\mathrm{Lsc}+\mathrm{Lv}+\mathrm{Lm}, \mathrm{Ls}=\mathrm{Lss}+\mathrm{Lsc}, \mathrm{Ltot}=\mathrm{L}+\mathrm{Qp}, \mathrm{QFL} \% \mathrm{Q}=100 \times \mathrm{Q} /(\mathrm{Q}+\mathrm{F}+\mathrm{L}), \mathrm{QFL} \% \mathrm{~F}=100 \times \mathrm{F} /(\mathrm{Q}+\mathrm{F}+\mathrm{L}), \mathrm{QFL} \% \mathrm{~L}=100 \times \mathrm{L}(\mathrm{Q}+\mathrm{F}$ $+\mathrm{L}), \mathrm{QmFLtot} \% \mathrm{Qm}=100 \times \mathrm{Qm} /(\mathrm{Qm}+\mathrm{F}+\mathrm{Ltot}), \mathrm{QmFLtot} \% \mathrm{~F}=100 \times \mathrm{F} /(\mathrm{Qm}+\mathrm{F}+\mathrm{Ltot}), \mathrm{QmFLtot} \% \mathrm{~F}=100 \times \mathrm{LtotP} /(\mathrm{Qm}+\mathrm{F}+\mathrm{Ltot}), \mathrm{LmLvLs} \% \mathrm{Lm}=100 \times \mathrm{Lm} / \mathrm{elm}+\mathrm{Lv}$ + Lss + Lsc), $\mathrm{LmLvLs} \% \mathrm{Lv}=100 \times \mathrm{Lv} /(\mathrm{Lm}+\mathrm{Lv}+\mathrm{Lss}+\mathrm{Lsc}), \mathrm{LmLvLs} \% \mathrm{Ls}=100 \times \mathrm{Ls} /(\mathrm{Lm}+\mathrm{Lv}+\mathrm{Lss}+\mathrm{Lsc})$. Abbreviations are defined in Table 2

A

Facies 3

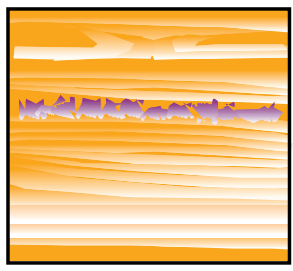

Section 960A-38R-2
B

Facies 3

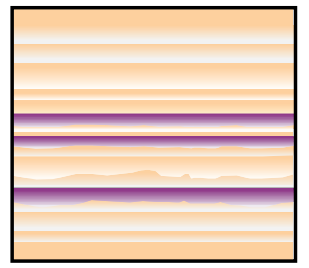

Section 960 A-46R-2
C

Facies 7

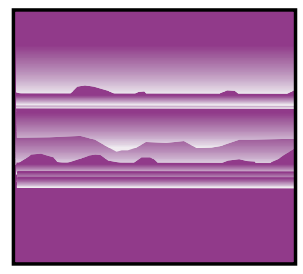

Section 960 A-57R-1
D

Facies 2

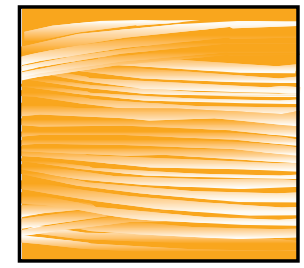

Section 959D-74R-2
E

Facies 7, 8, and 3

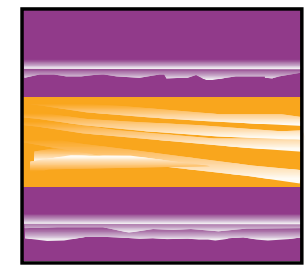

Section 959D-75R-1

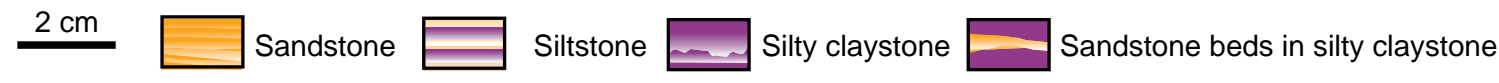

Figure 3. Dominant lithofacies types or successions occurring in Albian rift to early postrift stage. A. Laminated (convoluted) sandstone (Facies 3). B. Laminated siltstone (Facies 3). See also core-face photograph of interval 159-960A-46R-2, 34-49 cm, on CD-ROM (back pocket, this volume). C. Laminated silty claystone (Facies 7). See also core-face photograph of interval 159-960A-57R-1, 75-89 cm, on CD-ROM. D. Hummocky cross-bedded sandstone (Facies 2). See also core-face photographs of interval 159-959D-74R-2, 41-58 cm, on CD-ROM. E. Horizontally laminated (Facies 3) to cross-laminated (Facies 1) sandstone beds intercalated with laminated silty claystone (Facies 7, 8). See Table 1 for an interpretation of these specific facies.

\section{Transform to Passive Margin Transition During Late Coniacian to Early Eocene}

\section{Lithofacies}

At Site 960, the most prominent transition is between the Turonian-Santonian carbonate-cemented sandstones and limestones and the Eocene claystones. Also at Site 959, lower Coniacian sandy limestones are unconformably overlain by a condensed sequence of claystones containing phosphatic debris and hardgrounds (Facies 8; Fig. 7A). This condensed hemipelagic interval is overlain by a thick sequence of organic-rich black claystones with pyrite concentrations and Zoophycos burrows (Facies 8; Fig. 7B). The sequence is bracketed by the upper Santonian sediments below and the middle upper Paleocene strata above (Shipboard Scientific Party, 1996). At Site 961, lithologic preservation during Late Cretaceous to early Eocene was relatively poor; however, a transition from laminated sandy to clayey siltstone (Facies 7) to silty claystone (Facies 8) with pyrite and glau- 

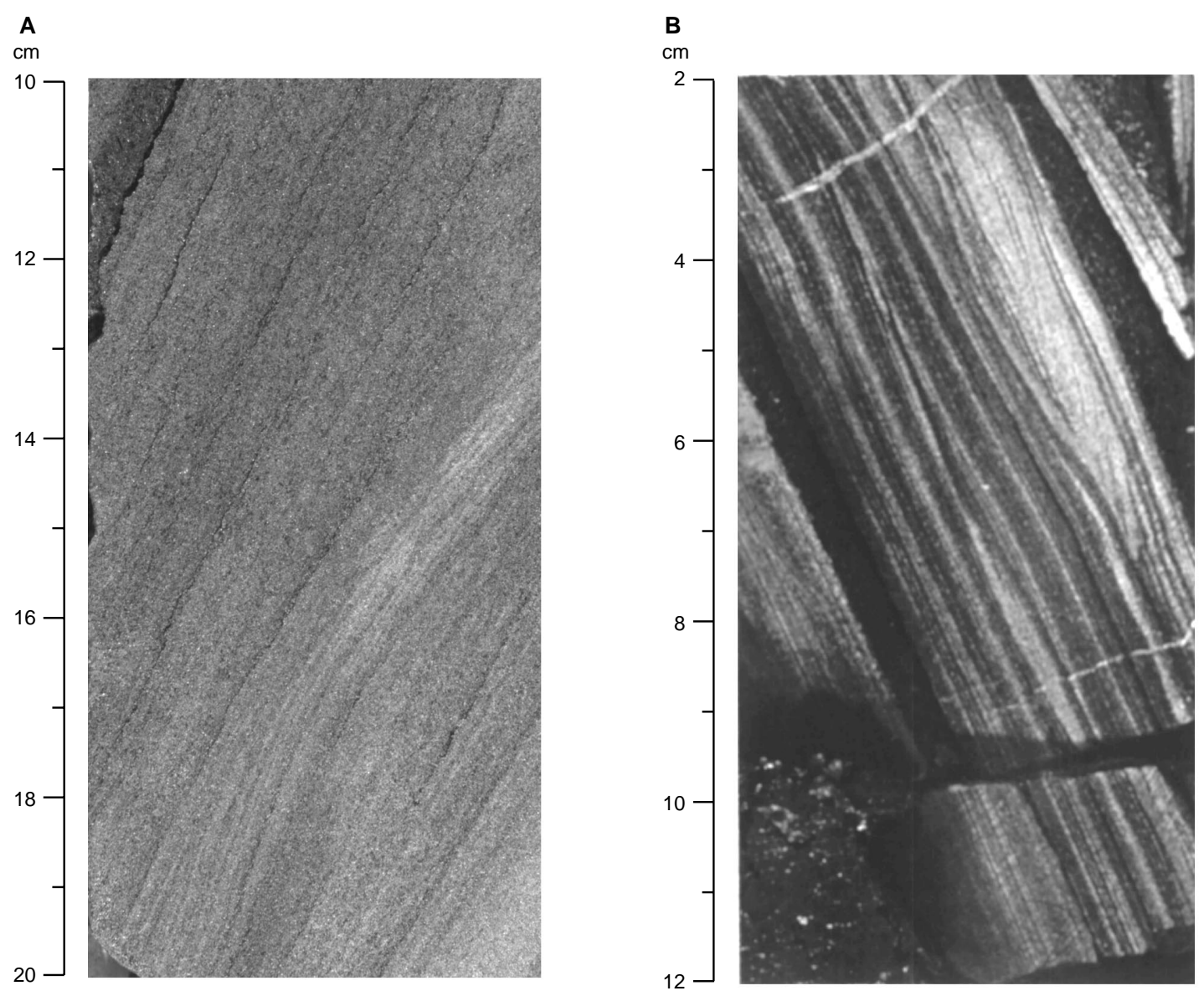

Figure 4. Typical sandy bedforms of Leg 159 sediments. A. A typical hummocky cross-bedded sandstone (Facies 2), interval 159-959D-77R-1, 8-20 cm. See also core-face photograph of interval 159-959D-77R-1, 5-23 cm, on CD-ROM (back pocket, this volume). B. A typical turbiditic bed with isolated ripples in quartz-sand limestone (Facies 12), interval 159-962D-23R-CC, 2-12 cm. See also core-face photograph of interval 159-962D-23R-CC, 0-12 cm, on CD-ROM.

A

Facies 10

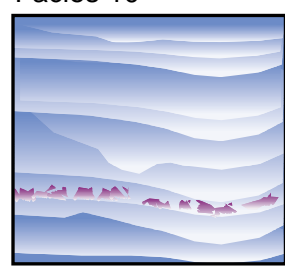

Section 960A-35R-1 $2 \mathrm{~cm}$

\section{B} Facies 9

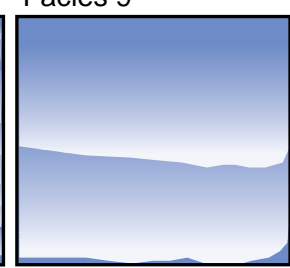

Section 960A-31R-1

Grainstone/ packstone
C

Facies 11

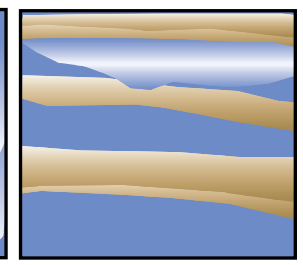

Section 960A-22R-2

Quartz sand grainstone

Figure 5. Dominant lithofacies types or successions occurring in Cenomanian-Coniacian continent/ocean transform stage. A. Skeletal grainstone with mudstone intraclasts (Facies 10). See also core-face photograph of interval 159-960C-25X-1, 112-123 cm, on CD-ROM (back pocket, this volume). B. Graded to crudely bedded skeletal packstone (Facies 9). See also core-face photograph of interval 159-960A-22R-1, 26-40 cm, on CD-ROM. C. Laminated quartz-sand grainstone and floatstone (Facies 11). See Table 1 for an interpretation of these specific facies.

conite concentrations is recorded (see intervals 159-961A-21R-2, 30-44 cm; 23R-1, 40-53 cm; on CD-ROM, back pocket, this volume). The discontinuous, irregular siltstone laminae are dominated by angular to subangular monocrystalline quartz, some fresh feldspar, and some mica flakes (Sample 159-961A-23R-1, 50-51 cm, on CD-ROM). Beginning in the late Paleocene, calcareous pelagic sedimentation was renewed in the Leg 159 area. Also Paleocene porcellanites (Facies 15; Fig. 7C) are found at Sites 959 and 961. Beginning in the lower Eocene, claystones enriched in, or even dominated by, palygorskite were recovered at Sites 960 (see interval 159-960A19R-1, 27-41 cm, on CD-ROM), 961, and 962.

\section{Interpretation}

Termination of the carbonate sedimentation implies a change in the depositional environment, and the crest of the marginal ridge appears to have undergone a phase of very rapid subsidence. At Site 961, this subsidence is marked by a transition from irregularly laminated sandy to clayey siltstone to deeper shelf claystone containing glauconite concentrations. The discontinued, irregular lamination in siltstone infers rapid deposition from fine-grained turbidity currents or reworking by winnowing bottom currents on the shelf (e.g., Pickering et al., 1986). The condensed section and a partially dysaerobic regime were probably a result of damming by the marginal ridge that restricted the depositional basins. Claystones dominate the sedimentation at Site 959 into the upper Paleocene, and at Site 960 until the 

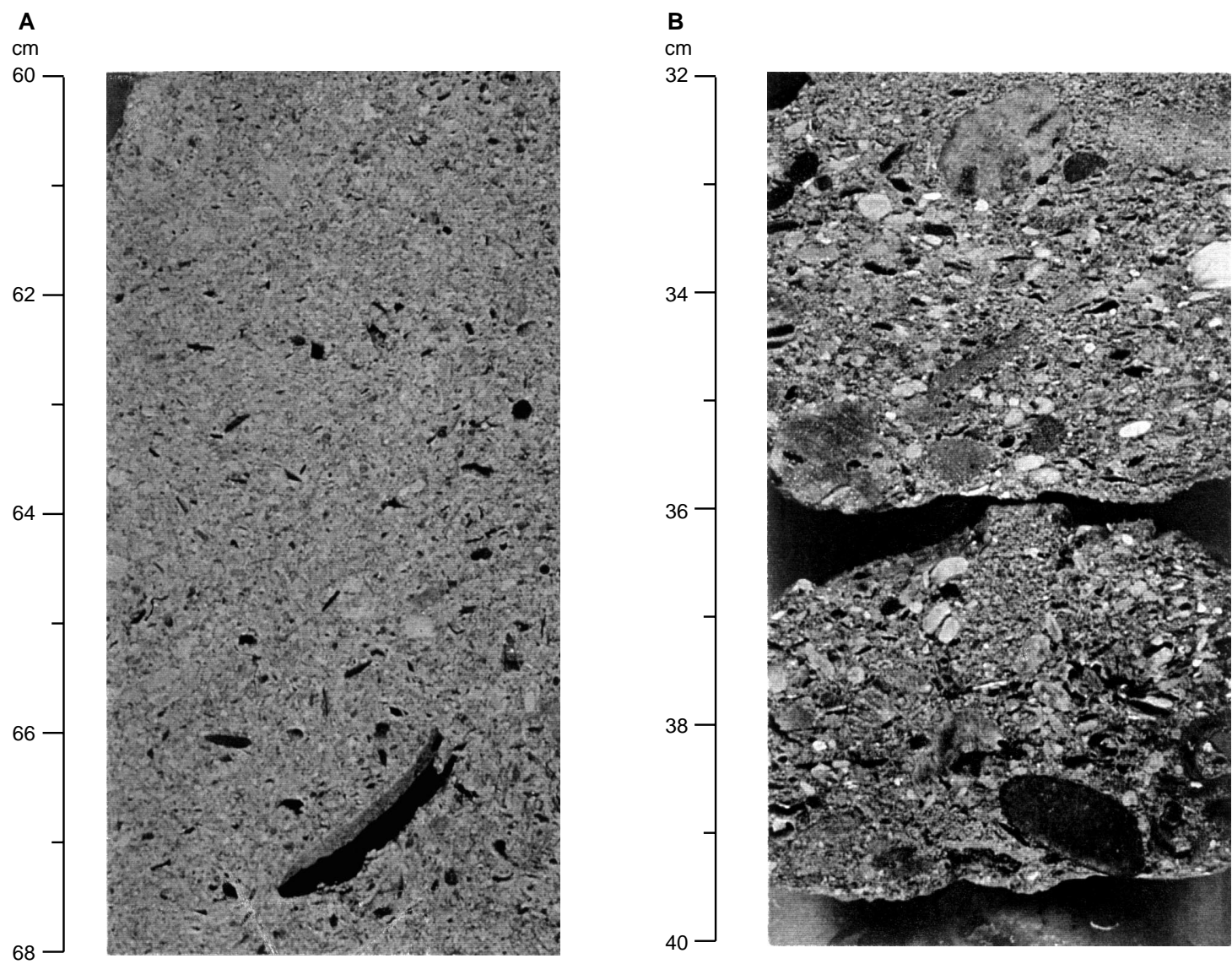

Figure 6. Typical structures and textural patterns in carbonate sediments of Leg 159. A. Skeletal grainstone with quartz sand (Facies 10), interval 159-960A25R-1, 58-68 cm. See also core-face photograph of interval 159-960A-25R-1, 56-70 cm, on CD-ROM (back pocket, this volume). B. Graded to crudely bedded quartz-sand packstone (Facies 9), interval 159-960A-22R-1, 30-40 cm. See also core-face photograph of interval 159-960A-22R-1, 26-40 cm, on CD-ROM.

A

Facies 8

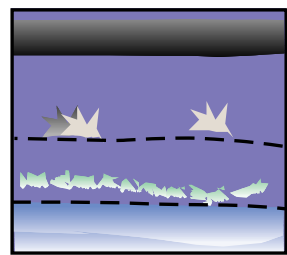

Section 960A-21R-1

$2 \mathrm{~cm}$

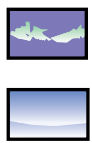

B

Facies 8

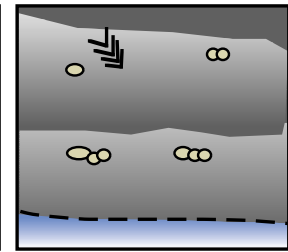

Section 959D-43R-1 Section 959D-9R-1

Claystone with phoshatic debris

Grainstone/ packstone
C Facies 15

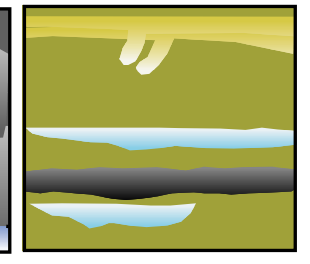

Porcellanite

Black claystone with pyrite

Figure 7. Dominant lithofacies type or successions occurring in late Coniacian-early Eocene transform to passive margin transition. A. Claystone (Facies 8) with phosphatic debris. See also core-face photograph of interval 159-960A-19R-1, 27-41 cm, on CD-ROM (back pocket, this volume). B. Black claystone (Facies 8). C. Porcellanite (Facies 15). See Table 1 for an interpretation of these specific facies.

Eocene. Sedimentation shifted to pelagic and hemipelagic, with intervals of siliceous material accumulating at Sites 959 and 961. Overall, these relatively thin stratigraphic records indicate that much of the area underwent sediment starvation through the Late Cretaceous.

\section{SEDIMENT COMPOSITION}

Cretaceous sandy layers cored during Leg 159 were petrographically analyzed to characterize their composition and provenance. Facies analysis is integrated with provenance analysis to better achieve possible source-rock composition, transport to the site of deposition, and the depositional environments. Furthermore, a transform margin can be taken as a specific tectonic environment characterized by modal sandstone composition (cf. Valloni and Maynard, 1981; Valloni, 1985). The most common framework grains include monocrystalline quartz, plagioclase, and potassium feldspar. Petrographic analyses show that the sandstones and sand-rich limestones are quartzose, with some spread of values toward the lithic-rich and quartz-rich ends of the diagrams (Fig. 8). Polycrystalline quartz was distinguished in the upper Albian successions at Site 959 (Table 2). Metamorphic rock fragments (see Sample 159-959D-76R-2, 0-3 cm on CD-ROM, back pocket, this volume) are concentrated in Albian successions at all sites, especially Sites 959 and 960 . These fragments must be derived from uplifted crystalline igneous and metamorphic terranes of continental origin. Typically, the nearby West African Craton is dominated by the Proterozoic, metamorphosed, Birimian and Tarkwaian volcanic 
Figure 8. Provenance analysis of Leg 159 sediments by sites. A. Ternary plot of QFL data. B. Ternary plot of QmFLtot data. C. Ternary plot of LmLvLs data. See Tables 1 and 2 for counted and recalculated parameters, respectively. Provenance domains after Dickinson et al. (1983).
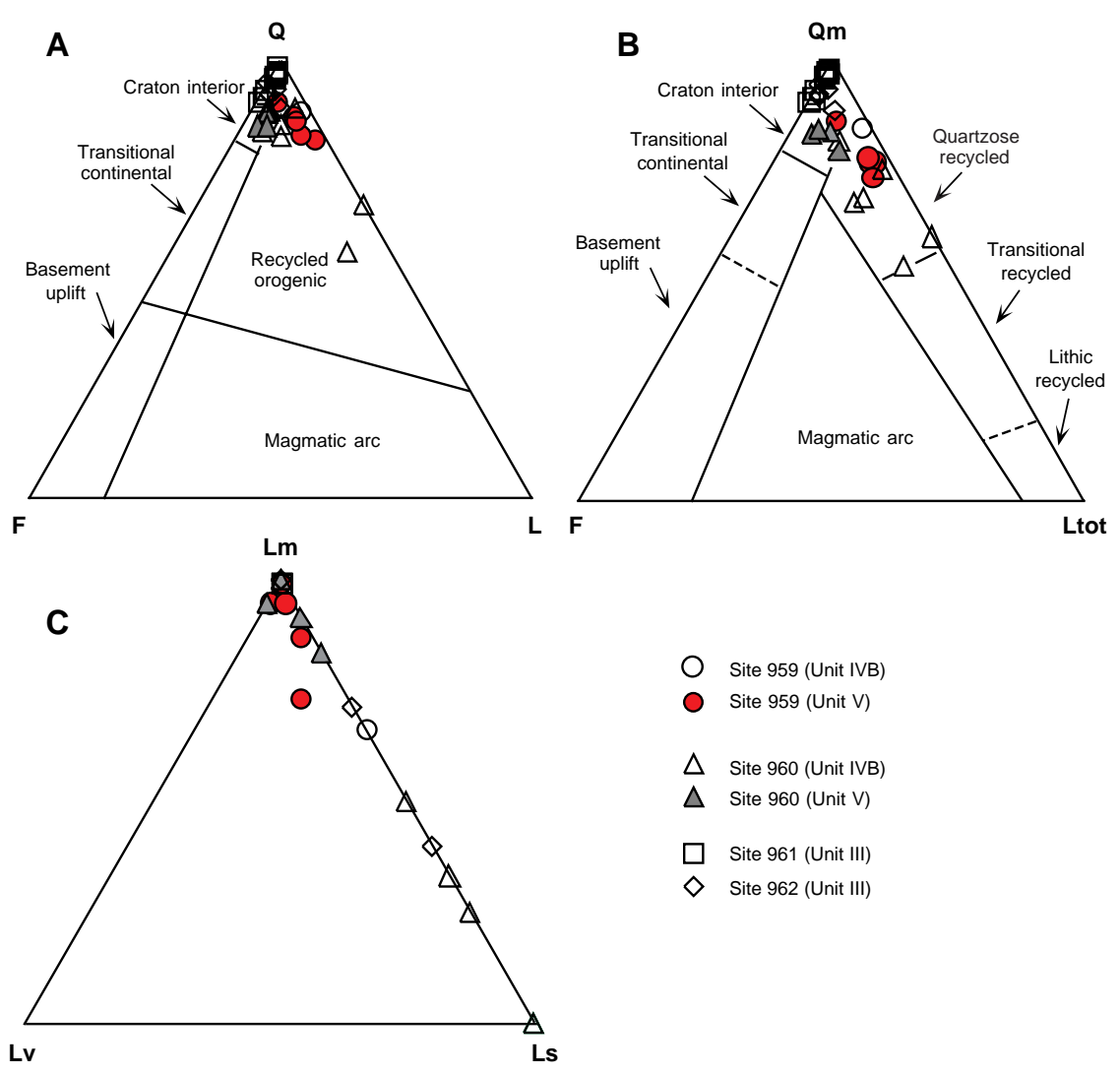

and sedimentary rocks and associated granite-gneiss terranes (Wright, 1985; Boher et al., 1992), which can be prominent source rocks. They covered what is now Ghana and Ivory Coast and much of Upper Volta, and they may also be represented on the other side of the Atlantic in northeast Brazil and Guyana, within the São Francisco Craton (cf. Bernasconi, 1983; Delgado et al., 1994). Sedimentary lithic fragments, both siliciclastics and carbonate, are present in TuronianSantonian carbonate-rich sediments (Table 2). These sediments also contain some metamorphic lithic fragments (Pl. 1, Figs. 1, 2). Rounded clasts reflect some recycling. Angular quartz and fresh feldspars in the lowermost portions of Site 960 (Pl. 1, Figs. 4, 5) indicate primary, proximal source.

Leg 159 sand composition reflects changes in provenance along the development of transform margin. Stable cratons of low relief generally produce quartzose sands from granite-gneiss basement and recycling of earlier strata, further transported to continental margins. Basement uplifts are areas of high relief along rifts and strike-slip zones. They produce sands that are dominantly quartzo-feldspathic, whereas lithic-poor sands are generally deposited in extensional basins (Fig. 8; Unit V of Sites 959 and 960). The Leg 159 transform margin seems to contain detritus that is dominantly quartzo-lithic, with few if any volcanic grains. Quartz and sedimentary lithic clasts dominate. The occurrence of sedimentary lithic grains reflects some recycling. Metamorphosed rock fragments indicate also that some levels of metamorphic basement were uplifted along the transform boundary during the Turonian-Santonian (Fig. 8B; Subunit IVB of Sites 959 and 960). Calculating the percentage of sedimentary rock fragments (Lss + Lsc) and metamorphic grains (Lm) up through a sequence seems to show trends relating to some source-area uplift or reworking of preexisting sedimentary units (Fig. 8C). An increase in Lss, and especially in Lsc, in Turonian-Santonian calcareous sandstones likely is caused by erosion and uplift of a reefal shelf. Also, some amount of sedimentary recycling of previously deposited sediments occurred related to this marginal ridge uplift and erosion.

\section{TECTONO-EUSTATIC CYCLES IN A RIFT/ TRANSFORM MARGIN TRANSITION}

Schematic block diagrams (Fig. 9) summarize the sediment sources and depositional environments of the Côte d'Ivoire-Ghana Transform Margin within the framework of the main tectonic evolution stages (after Shipboard Scientific Party, 1996). Figure 10 shows a synthesis of cyclicity in sedimentation and inferred relative changes of the sealevel curve for a margin created by transform tectonics. The Cretaceous rift to transform margin deposits document the early evolution of an active transform margin and its transition to a passive margin, thus showing a second-order, tectono-eustatic cycle of 10-50-m.y. duration (cf. Miall, 1990; Vail et al., 1991). This transition implies progressive subsidence of the margin related to the continental breakup and overall, relative rise in sea level. Within that transition, sedimentary characteristics also seem to express shorter duration, third-order (1-10 Ma) cycles of relative sea level, most probably controlled by regional tectonics. An individual third-order cycle is generally coarsening upward or at least corresponds to superimposed depositional environments, showing a progressively shallowing depositional interface (Haq, 1991).

The late Albian restricted intracontinental sedimentation (Fig. 9; Stage I) was followed by a progressive marine transgression (Fig. 9; Stage II) and deposition that terminates in an unconformity defining one total third-order cycle (Fig 10). The overlying carbonate deposits of Turonian to Santonian age derived from shallow reefs indicate a beginning of marginal ridge uplift (Fig. 9; Stage III). A significant siliciclastic component accompanied by carbonate sedimentation indicates mixed, reworked sediment sources, supposed further uplift of the marginal ridge and reduction of sediment accommodation space, and thus termination of the next third-order cycle (Fig. 10). The coeval carbonate and siliciclastic sedimentation could mean a maximum uplift of the marginal ridge, thought to be related to the thermal effect of the passage of a spreading ridge south of the margin. Times 
I. End of rifting during Albian

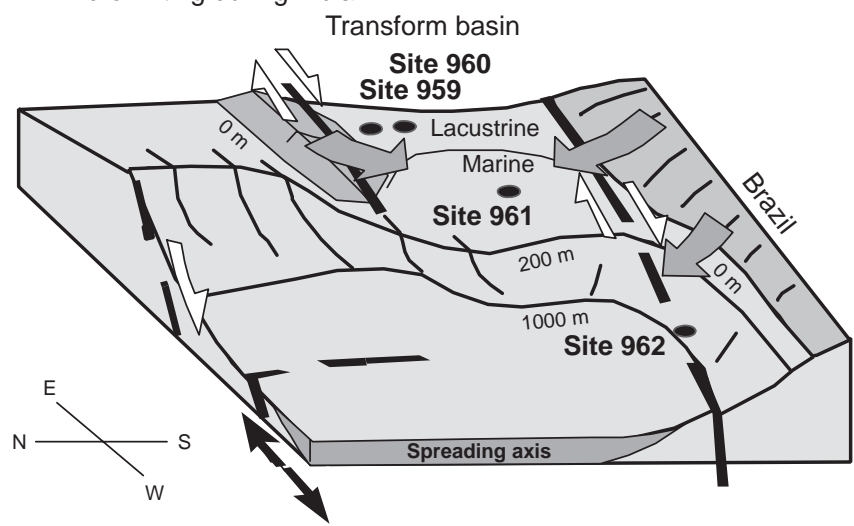

II. Late Albian early post-rift stage Transform basin

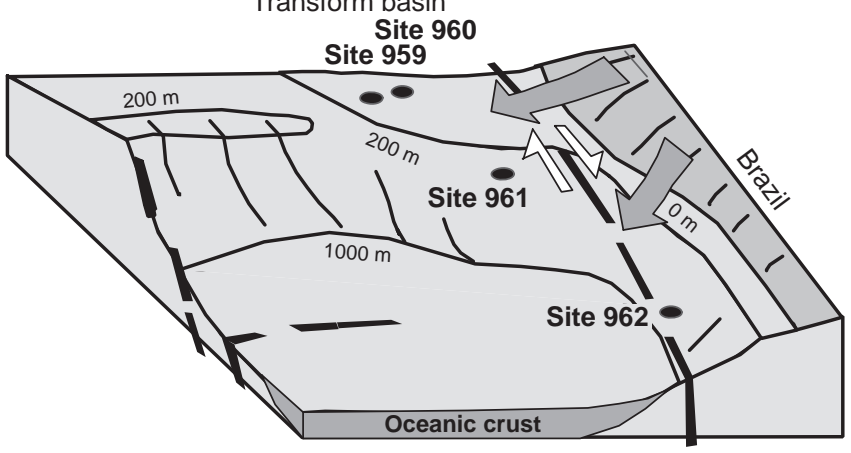

III. Continent/ocean transform stage (Cenomanian-Coniacian) Transform basin

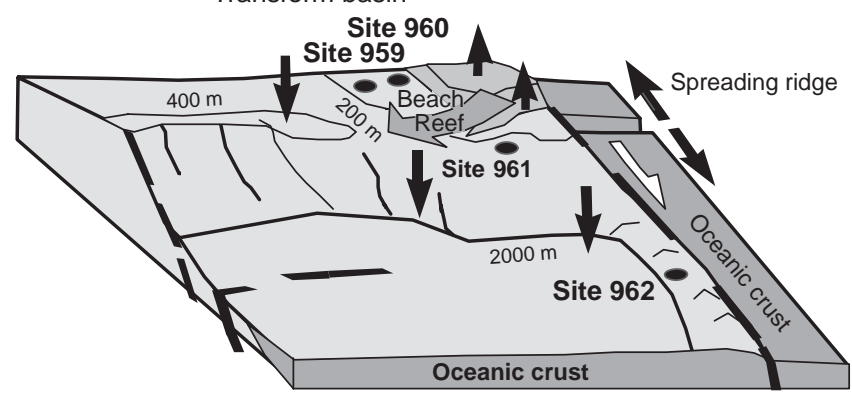

IV. Transform to passive margin transition (late Coniacianearly Eocene)

Restricted basin(dysaerobic)

Palygorskite clays-diatomites

Hardgrounds - black claystones Site 960

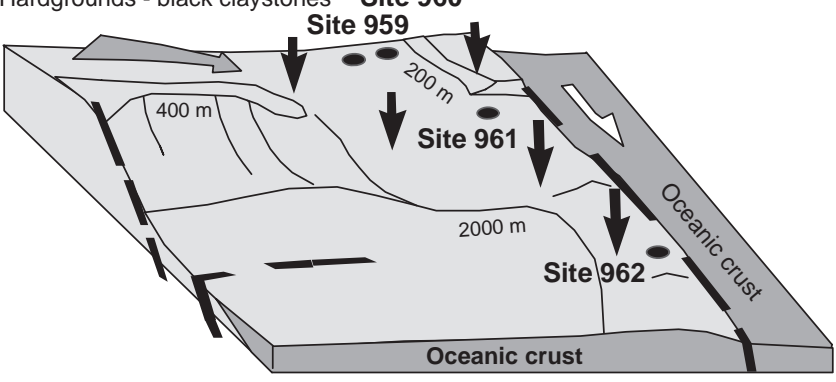

Figure 9. Schematic block diagrams showing the main tectonic evolutionary stages (after Shipboard Scientific Party, 1996), thermally triggered uplift and subsidence (black arrows) and related sediment sources (gray arrows), and depositional basins of the Côte d'Ivoire-Ghana Transform Margin viewed from the northwest.

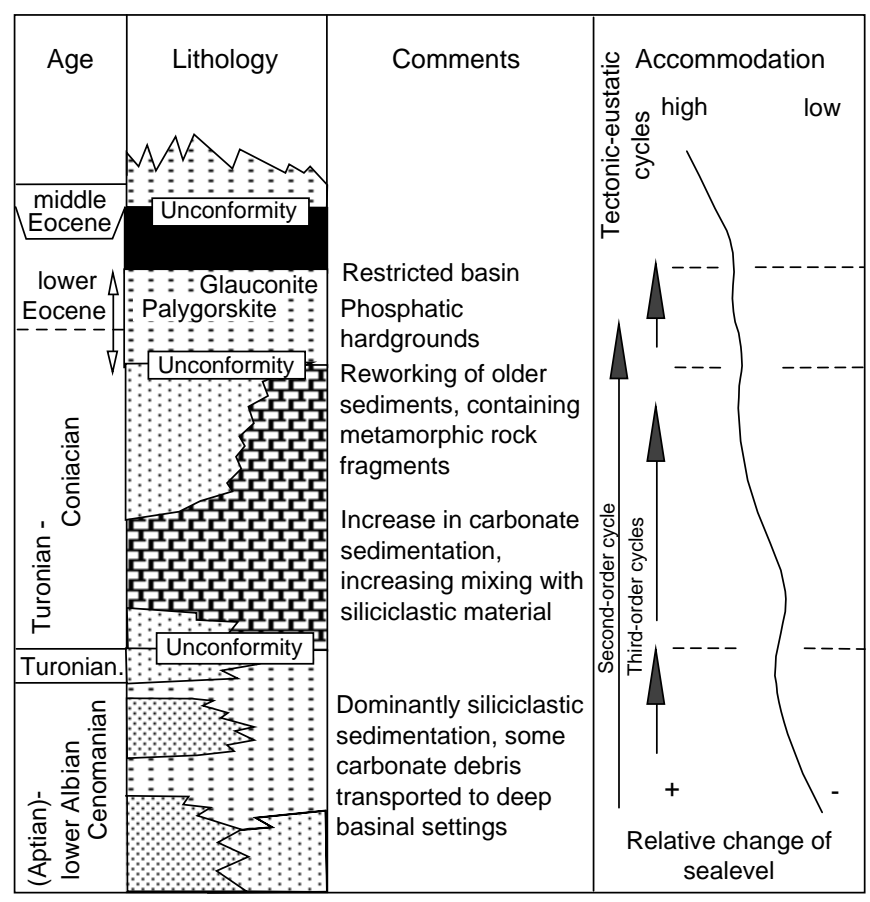

Figure 10. Synthesis of tectono-eustatic cycles, accommodation, and relative change of sea level for the Côte d'Ivoire-Ghana Marginal Ridge during the Cretaceous. See Figure 2 for a key to lithologies.

of marginal ridge uplift also represent times of low relative sea level. Models of the passage of a spreading ridge along a transform margin (e.g., Todd and Keen, 1989) or seismic stratigraphic studies from other margins (e.g., Exmouth Plateau; Lorenzo and Vera, 1992) suggest that major amounts of uplift may result from the juxtaposition of hot, young, oceanic crust and older continental crust. The next cycle over the sandy limestones is recorded by a condensed section of claystones containing hardgrounds deposited within a time period of more than 10 m.y. (Fig. 9; Stage IV). In a deeper setting, this condensed interval is overlain by a sequence of organic-rich black claystones. This deposition was promoted by slightly diminishing accommodation space and partial damming of the basin by the marginal ridge (Fig. 10) and deposition of palygorskite clay, which requires high salinities for its formation (Pletsch, Chap. 15, this volume). Stage IV (Fig. 9) started the progressive deepening of the basin related to thermal subsidence, accompanied by a shift to a normal passive margin tectonic setting.

\section{CONCLUSIONS}

Drilling of the Côte d'Ivoire-Ghana Marginal Ridge represents the first detailed drilling survey of a transform margin. In this study, the investigations were intended to achieve an understanding of the tectonic processes and to provide sedimentary indicators that may be related to the formation of transform margins.

1. Intracontinental sedimentation with lacustrine and deltaic deposits was followed by deposition of storm-influenced shelf sandstones. These are unconformably overlain by pelagic and periplatformal carbonate deposits from shallow reefs, marking an apparent episode of marginal ridge uplift. These are disconformably overlain by a condensed section of claystones with hardgrounds. After the late Paleocene, sedimentation was pelagic and hemipelagic.

2. Metamorphosed rock fragments indicate that basement terranes were uplifted during the Santonian-Turonian along the transform boundary. Sedimentary rock fragments and metamorphic grains up 
through a sequence seem to show trends relating to source-area uplift and partial reworking of preexisting sedimentary units. This uplift in a source area exposes deeper levels to erosion.

3 . This study identified one second-order cycle (10-50 Ma) of relative sea-level change, related to the continental breakup of a margin, and three third-order (1-10 Ma) cycles of tectono-eustatic controlled relative change of sea level in a margin created by transform tectonics. These third-order cycles relate to a complex history of thermally triggered uplift and subsidence resulting from tectonic stress release along the continent/continent transform and caused by the proximity of a passing oceanic spreading center.

\section{ACKNOWLEDGMENTS}

I would like to thank the Ocean Drilling Program for providing samples for this study, and especially the colleagues from the Leg 159 shipboard sedimentologists group and Jean Mascle for helpful discussions regarding the sediment interpretations and tectonic aspects. Michel Guiraud, William Heins, and Mark Johnsson provided constructive and helpful reviews that substantially improved the original manuscript. Participation in Leg 159 and this research was supported by the Natural Sciences and Technical Research Council of the Academy of Finland.

\section{REFERENCES}

Basile, C., Brun, J.P., and Mascle, J., 1992. Structure et formation de la marge transformante de Côte d'Ivoire-Ghana: apports de la sismique réflexion et de la modélisation analogique. Bull. Soc. Geol. Fr., 163:207216.

Basile, C., Mascle, J., Popoff, M., Bouillin, J.P., and Mascle, G., 1993. The Côte d'Ivoire-Ghana Transform Margin: a marginal ridge structure deduced from seismic data. Tectonophysics, 222:1-19.

Ben-Avraham, Z., Almagor, G., and Garfunkel, Z., 1979. Sediments and structure of the Gulf of Eilat (Aqaba)-northern Red Sea. Sediment. Geol., 23:239-267.

Bernasconi, A., 1983. The Archaean terranes of central eastern Brazil: a review. Precambrian Res., 23:107-131.

Boher, M., Abouchami, W., Michard, A., Albarede, F., and Arndt, N.T., 1992. Crustal Growth in West Africa at 2.1 Ga. J. Geophys. Res., 97:345-369.

Delgado, I.de M., Pedreira, A.J., and Thorman, C.H., 1994. Geology and mineral resources of Brazil: a review. Int. Geol. Rev., 36:503-544.

Dickinson, W.R., 1970. Interpreting detrital modes of graywacke and arkose. J. Sediment. Petrol., 40:695-707.

Dickinson, W.R., Beard, L.S., Brakenridge, G.R., Erjavec, J.L., Ferguson, R.C., Inman, K.F., Knepp, R.A., Lindberg, F.A., and Ryberg, P.T., 1983. Provenance of North American Phanerozoic sandstones in relation to tectonic setting. Geol. Soc. Am. Bull., 94:222-235.

Friedman, G.M., 1971. Staining. In Carver, R.E. (Ed.), Procedures in Sedimentary Petrology: New York (Wiley-Interscience), 511-530.

Haq, B.U., 1991. Sequence stratigraphy, sea-level change, and significance for the deep sea. In Macdonald, D.I.M. (Ed.), Sedimentation, Tectonics and Eustasy: Sea Level Changes at Active Margins. Spec. Publ. Int. Assoc. Sedimentol., 12:3-39.

Honnorez, J., Villeneuve, M., and, Mascle, J. 1994. Old continent-derived metasedimentary rocks in the Equatorial Atlantic: an acoustic basement outcrop along the fossil trace of the Romanche transform fault at $6^{\circ} 30^{\prime} \mathrm{W}$. Mar. Geol., 117:237-251.
Ingersoll, R.V., Bullard, T.F., Ford, R.L., Grimm, J.P., Pickle, J.D., and Sares, S.W., 1984. The effect of grain size on detrital modes: a test of the GazziDickinson point-counting method. J. Sediment. Petrol., 54:103-116.

Lorenzo, J.M., and Vera, E.E., 1992. Thermal uplift and erosion across the continent - ocean transform boundary of the southern Exmouth Plateau. Earth Planet. Sci. Lett., 108:79-92.

Mascle, J., and Blarez, E., 1987. Evidence for transform margin evolution from the Côte d'Ivoire-Ghana continental margin. Nature, 326:378-381.

Mascle, J., Guiraud, M., Basile, C., Benkhelil, J., Bouillin, J.P., Cousin, M., and Mascle, G., 1993. La marge transformante de Côte d'Ivoire-Ghana: premiers résultats de la campagne Equanaute (Juin 1992) [The Côte d'Ivoire-Ghana transform margin: preliminary results from the Equanaute cruise (June 1992)]. C. R. Acad. Sci. Ser. 2, 316:1255-1261.

Mascle, J., Lohmann, G.P., Clift, P.D., et al., 1996. Proc. ODP, Init. Repts., 159: College Station, TX (Ocean Drilling Program).

Miall, A.D., 1990. Principles of Sedimentary Basin Analysis. Second Edition: New York (Springer-Verlag).

Myrow, P.M., and Southard, J.B., 1991. Combined-flow model for vertical stratification sequences in shallow marine storm deposits. J. Sediment. Petrol., 61:202-210.

Nottredt, A., and Kreisa, R.D., 1987. Model for the combined-flow origin of hummocky cross-stratification. Geology, 15:357-361.

Pickering, K., Stow, D., Watson, M., and Hiscott, R., 1986. Deep-water facies, processes and models: a review and classification scheme for modern and ancient sediments. Earth-Sci. Rev., 223:75-174.

Pickering, K.T., Hiscott, R., and Hein, F.J., 1989. Deep-marine Environments: Clastic Sedimentation and Tectonics: London (Unwin Hyman).

Piper, D.J.W., and Stow, D.A.V., 1991. Fine-grained turbidites. In Einsele, G., and Seilacher, A. (Eds.), Cyclic and Event Stratification: New York (Springer Verlag), 360-376.

Pitman, W.C., III, and Andrews, J.A., 1985. Subsidence and thermal history of small pull-apart basins. In Biddle, K.T., and Christie-Blick, N. (Eds.), Strike-slip Deformation, Basin Formation, and Sedimentation. Spec. Publ-Soc. Econ. Paleontol. Mineral., 37:45-49.

Scholle, P.A., Bebout, D.G., and Moore, C.H. (Eds.), 1983. Carbonate Depositional Environments. AAPG Mem., 33.

Shipboard Scientific Party, 1996. Principal results. In Mascle, J., Lohmann, G.P., Clift, P.D., et al., Proc. ODP, Init. Repts., 159: College Station, TX (Ocean Drilling Program), 297-314.

Stow, D.A.V., and Shanmugam, G., 1980. Sequences of structures in finegrained turbidites: comparison of recent deep-sea and ancient flysch sediments. Sediment. Geol., 25:23-42.

Todd, B.J., and Keen, C.E., 1989. Temperature effects and their geological consequences at transform margins. Can. J. Earth Sci., 26:2591-2603.

Tucker, M.E., and Wright, P.V., 1990. Carbonate Sedimentology: Oxford, (Blackwell Sci. Publ.).

Vail, P.R., Audemard, F., Bowman, S.A., Eisner, P.N., and Perez-Cruz, G., 1991. The stratigraphic signatures of tectonics, eustasy, and sedimentology-an overview. In Einsele, G., Ricken, W., and Seilacher, A. (Eds.), Cycles and Events in Stratigraphy: Berlin (Springer), 617-659.

Valloni, R., 1985. Reading provenance from modern marine sands. In Zuffa, G.G. (Ed.), Provenance of Arenites. NATO ASI Ser. C, 148:309-332.

Valloni, R., and Maynard, J.B., 1981. Detrital modes of recent deep-sea sands and their relation to tectonic setting: a first approximation. Sedimentology, 28:75-83.

Wright, J.P., 1985. Geology and Mineral Resources of West Africa: London (Unwin).

Date of initial receipt: 24 September 1996

Date of acceptance: 12 March 1997

Ms 159SR-009 


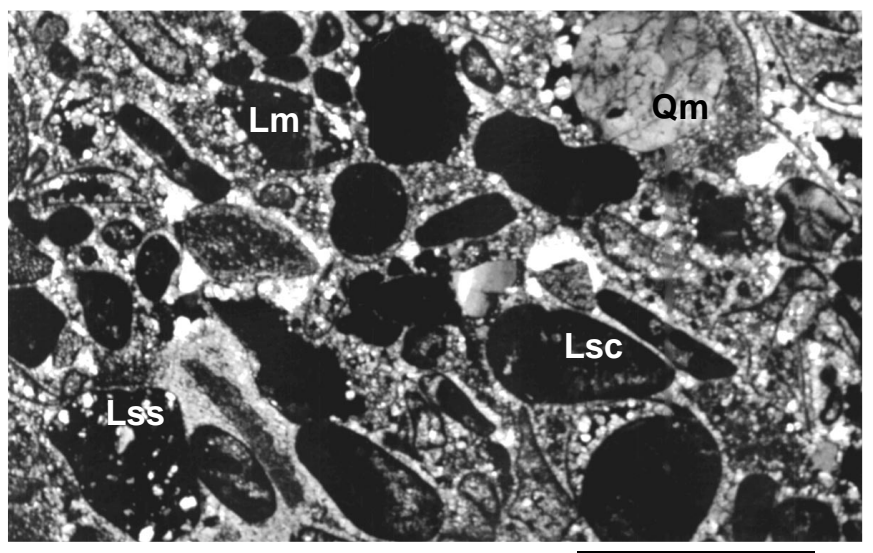

1

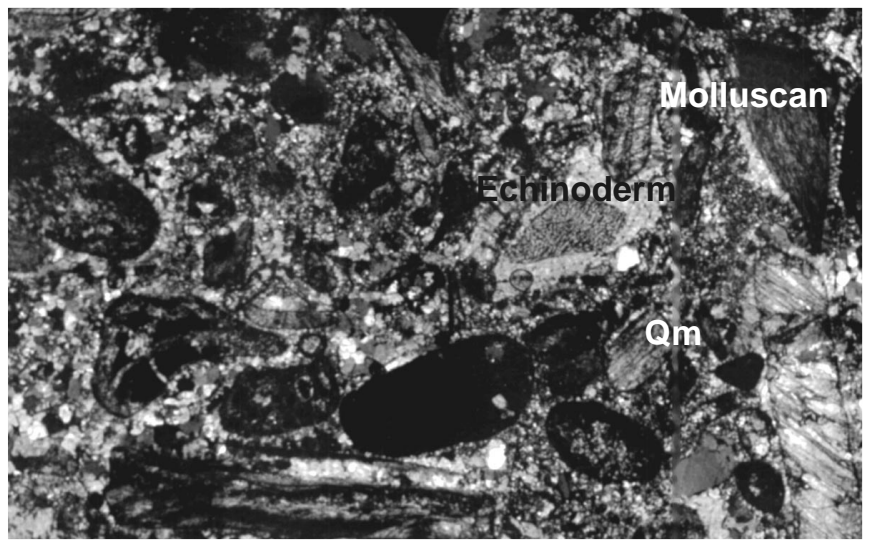

3

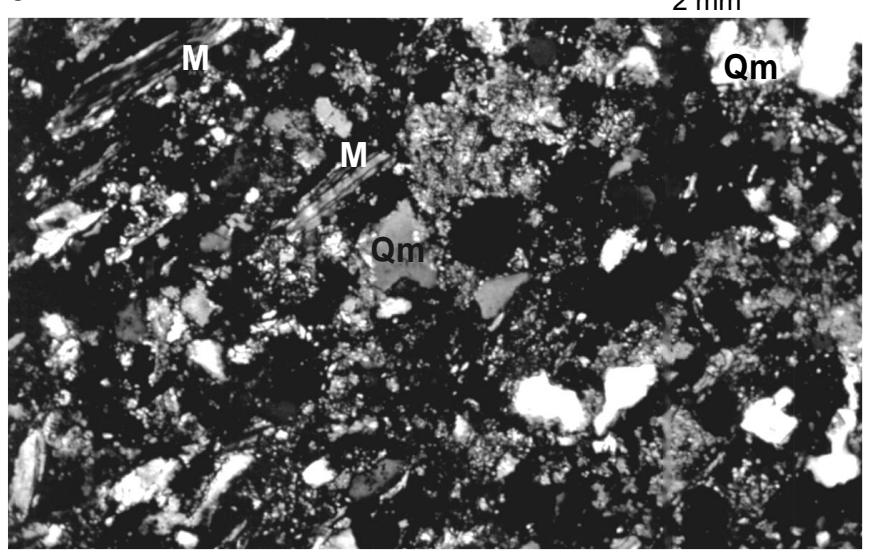

5

$2 \mathrm{~mm}$

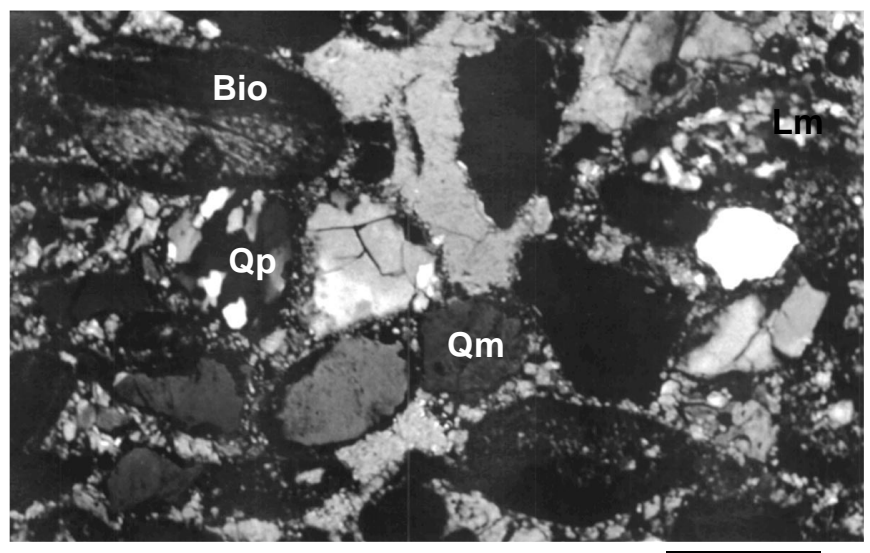

2

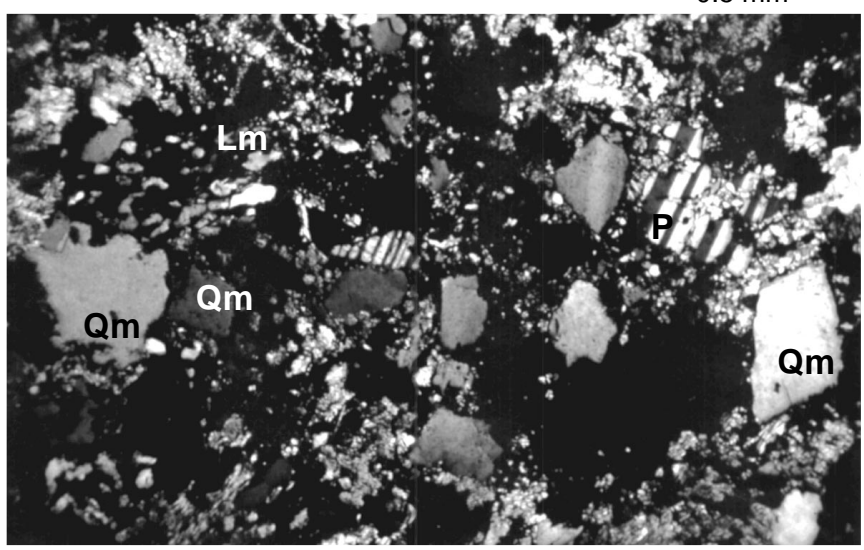

4

$0.5 \mathrm{~mm}$

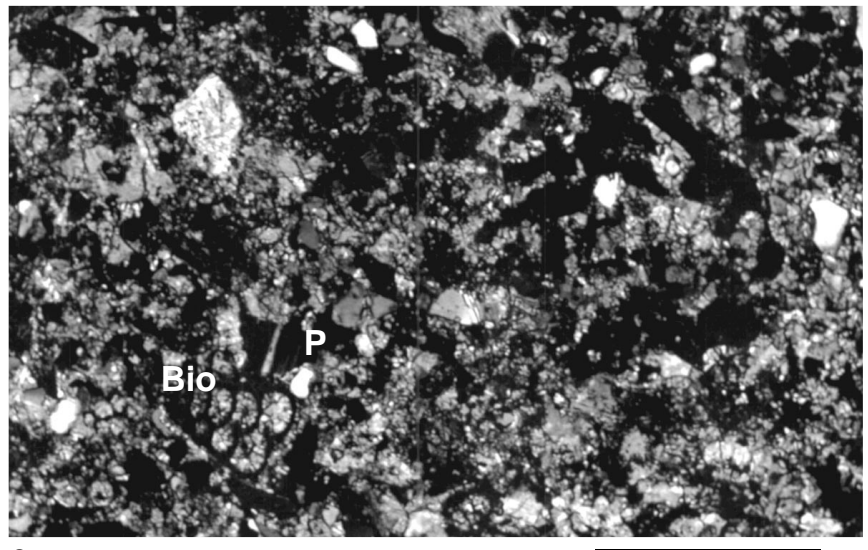

6

$2 \mathrm{~mm}$

Plate 1. Photomicrographs of sandstones and quartz sand limestones. All in cross-polarized light. 1. Rounded, monocrystalline (Qm) and metamorphic (Lm), siliciclastic sedimentary (Lss) and carbonate sedimentary lithic fragments (Lsc) in quartz-sand grainstone, Sample 159-960A-25R-2, 12-14 cm. Pore spaces are now filled by equant calcite cement. 2. Subangular to angular monocrystalline quartz (Qm), polycrystalline quartz (Qp), metamorphic lithic fragments (Lm), and skeletal fragments (Bio) in quartz-sand grainstone, Sample 159-960A-29R-3, 99-102 cm. 3. Skeletal grainstone containing molluscan fragments and echinoderm debris and a few monocrystalline quartz grains (Qm), Sample 159-960A-34R-1, 45-49 cm. Pore spaces are filled with calcite cement. 4. Subangular to angular monocrystalline quartz $(\mathrm{Qm})$, fresh plagioclase $(\mathrm{P})$, and metamorphic lithic fragments $(\mathrm{Lm})$ in medium-grained sandstone with micrite, Sample 159960A-37R-2, 49-52 cm. 5. Angular to subangular monocrystalline quartz $(\mathrm{Qm})$ and mica flakes $(\mathrm{M})$ in silty sandstone laminae. Carbonate occurs as a cement. Sample 159-960A-57R-1, 18-22 cm. 6. Limestone with quartz silt grains, plagioclase feldspar (P), and biogenic components (Bio) varying from planktonic foraminifers to bivalves, Sample 159-962D-36R-1, 4-7 cm. See also color photomicrographs of these identical samples on CD-ROM (back pocket, this volume). 